was commenced owing to the fact that wire nails had become very difficult to obtain in India, while the demand for packing cases, not least for army requirements, had increased enormously. Experiments were carried out to see how bamboo nails compared in holding power and other qualities with wire nails. The results of the tests are given; and sketches of largescale production of bamboo nails are included and the process of manufacture described.

Truly India is a fortunate country when her carefully supervised forest wealth enables her to confront one difficulty after another in times of dire stress through the work of her research officers. "It has been found", says the author, "that glued bamboo nails have a greater holding power than plain iron nails under a steady pull. But when shocks and transportation hazards are met with, their holding power diminishes". A small percentage of iron nails in a packing case is, therefore, necessary, especially for fixing the battens by clinching. It was found by actual tests that if 25 per cent iron nails and 75 per cent bamboo nails are used, a strong and serviceable box results, with a very considerable saving in costs of manufacture.

Another use of the bamboo has been found in the manufacture of pill or ointment boxes. Due to a shortage of metal, various substitutes have had to be used in the construction of containers; thus plywood containers have already been mentioned in NATURE (Ind.For. Leaf. 24 and 26). In Leaflet 39 (1943) Sultan Mohammed discusses the method of making from bamboo small pill and ointment boxes. Details of manufacture are given including some information on suitable coatings and linings. It is said that a fairly competent turner will find no difficulty in making these boxes with the aid of a lathe and ordinary tools. The description is of interest: "A bamboo container consists of a short length of bamboo (according to size of container required) which forms the walls of the body and lid of the container. The top and the bottom are made of thin three plywood and are provided with a locking device so that, if the top becomes loose due to climatic changes, the lid will still be held tight. The container is usually coated or painted, both inside and outside, with the object of retarding the absorption of moisture by sealing the pores of the bamboo, and to give a smooth clean finish."

\section{THE SCIENCE OF TOMATO GROWING}

$\mathrm{T}$

HE Cheshunt Experimental and Research Station has devoted its energies during war-time wholly to the elucidation of problems connected with food crops. Reports of investigations on the tomato occupy most of the twenty-eighth annual report, for 1942, of the Station (from the director, Dr. W. F. Bewley, The Nursery and Market Garden Industries' Development Society, Ltd., Turner's Hill, Cheshunt, Herts, 1943). Trials of the value of muriate of potash as against sulphate of potash resulted in a small, though scarcely significant, increase of crop in favour of the sulphate. No harmful foliage effects were observed when muriate was added, and it would seem that widespread doubts about the suitability of muriate of potash for the indoor tomato crop are now dispelled.

Much research has been devoted to diseases and pests of the tomato. Two varieties, Manx Marvel and Riverside, showed significant resistance to the wilt fungus Verticillium albo-atrum. Tomato stemrot caused by the fungus Didymella lycopersici caused widespread damage in 1942 to plants growing in the greenhouse and in the open. The disease appears to be carried from crop to crop on wire supports and strings, and may be controlled by suitable methods of hygiene. Experiments have also been made with fungicides as soil treatment, or applied as sprays and dusts to the plant, or as a protection of the 'collar', where infection normally occurs in greenhouse tomatoes.

Control of the wireworm pest by trapping with carrot bait gave rather disappointing results, and the elimination of these larvæ still remains one of the outstanding problems of pest control. Other negative results were obtained with sprays containing tartar-emetic and glycerine, advocated in the United States against the red-spider mite, and with potassium iodide solution for the prevention of damage by the root-knot eelworm.

Tomatoes in sand culture exhibited clear-cut symptoms when deprived of boron, manganese and magnesium. Omission of boron and magnesium from the culture solution produced marked stunting, and boron deficiency caused a leaf scorch similar to that sometimes associated with acute potassium starva. tion. Lack of magnesium induced a withering of the tips of leaflets, whereas the absence of manganese brought about a mottling of the leaf with uniform yellow specks. The report contains details of these and other investigations in the form of short papers by the authors of each subject for research.

\section{HYDROGEN EXCHANGE REACTIONS}

TN two papers (J. Chem. Soc., 361, 367; J943) H. V. A. Briscoe and co-workers describe the results of experiments on the interchange of hydrogen isotopes with complex salts, ammines of cobalt, and platinum and palladium being studied. The hydrogen of $\left[\mathrm{Co}\left(\mathrm{NH}_{3}\right)_{6}\right] \mathrm{Cl}_{3}$ and [Co $\left.\mathrm{en}_{3}\right] \mathrm{Cl}_{3}$ (en = ethylenediamine) undergoes isotope exchange with heavy water $\left(D_{2} O\right)$ in solutions, the interchange following a unimolecular law with a rate inversely proportional to the hydrogen ion concentration but practically independent of the ammine concentration or its deuterium content. The exchange mechanism is explained by assuming that the metal ammine group $M-\mathrm{NH}_{3}$ can ionize as an acid : $M-\mathrm{NH}_{3}=$ $M-\mathrm{NH}_{2}^{\prime}+\mathrm{H} \cdot$, this being parallel to the ionization of aquo-ammines, which form hydroxo-ammines. There is some evidence that the case of acid dissociation runs parallel with the stability of the ammines. The reaction mechanism is very fully discussed in the papers on the basis of the kinetics.

The rate of interchange of hydrogen isotopes with ammines of different metals decreases in the order $\mathrm{Co}>\mathrm{Pt}>\mathrm{Pd}$ and runs parallel to the complex ions. In the case of $\left[\mathrm{Pd}\left(\mathrm{NH}_{3}\right)_{4}\right] \mathrm{Cl}_{2}$ the interchange mechanism is complex; in addition to the acid dissociation mechanism, it reacts in acid solution by reversible dissociation of ammonia from the complex. This points to a continuous gradation between the two extreme types of ammonia complexes represented by the stable metal ammines of the $\left[\mathrm{Co}\left(\mathrm{NH}_{3}\right)_{6}\right] \mathrm{Cl}_{3}$ 